Research Article

\title{
Comparative Pharmacokinetics of Geniposidic Acid, Genipin-1- $\beta$ - Gentiobioside, Geniposide, Genipin, and Crocetin in Rats after Oral Administration of Crude Gardeniae Fructus and Its Three Processed Products Using LC-MS/MS
}

\author{
Xuejing Yang, ${ }^{1,2}$ Jin $_{\mathrm{Li}}{ }^{2,3}$ Xi Yang, ${ }^{2,3}$ Jun $\mathrm{He} \mathbb{D}^{2,3}$ and Yan-Xu Chang $\mathbb{C}^{2,3}$ \\ ${ }^{1}$ School of Pharmacy, Harbin University of Commerce, Harbin, Heilongjiang 150076, China \\ ${ }^{2}$ Tianjin State Key Laboratory of Modern Chinese Medicine, Tianjin University of Traditional Chinese Medicine, \\ 300193 Tianjin, China \\ ${ }^{3}$ Tianjin Key Laboratory of Phytochemistry and Pharmaceutical Analysis, Tianjin University of Traditional Chinese Medicine, \\ 300193 Tianjin, China \\ Correspondence should be addressed to Yan-Xu Chang; tcmcyx@126.com
}

Received 15 January 2020; Revised 1 April 2020; Accepted 16 April 2020; Published 7 May 2020

Academic Editor: Luigi Milella

Copyright (C) 2020 Xuejing Yang et al. This is an open access article distributed under the Creative Commons Attribution License, which permits unrestricted use, distribution, and reproduction in any medium, provided the original work is properly cited.

\begin{abstract}
The extract of Gardeniae Fructus (GF) with different processing methods processed the different medicinal properties and efficacy. Crude GF (CGF) could be processed into stir-frying GF (SGF), gancao mix-frying GF (GCGF), and ginger mix-frying GF (GIGF) in practice. An LC-MS/MS method was established for simultaneous quantification of geniposidic acid, geniposide, genipin-1$\beta$-gentiobioside, genipin, and crocetin in the rat plasma. The LLOQs for determination of all five components were $10 \mathrm{ng} / \mathrm{mL}$. The accuracies of intraday and interday were in the range of $91 \%-105 \%$. The recoveries of 5 analytes ranged from $81.0 \%$ to $114 \%$ with RSD less than $14 \%$. The results showed that the AUCs (area under the plasma concentration-time curve) and $C_{\max }$ (maximum plasma concentration) of geniposidic acid, genipin-1- $\beta$-gentiobioside, and geniposide after oral administration of the CGF extract were apparently higher than those after oral administration of other processed extracts. $C_{\max }$ of geniposide in plasma after administration of GIGF significantly decreased $(p<0.01)$. Genipin was not detected in rat plasma after administration of the GIGF extract, but it can be detected in plasma after administration of CGF, SGF, and GCGF extract. Furthermore, crocin I and crocin II were not detected in plasma samples. Crocetin had higher concentration in rat plasma versus lower contents in extract. It was demonstrated that the different processing methods might influence the pharmacokinetics of geniposidic acid, genipin-1$\beta$-gentiobioside, geniposide, genipin, and crocetin.
\end{abstract}

\section{Introduction}

According to processing theory of traditional Chinese medicine (TCM), medicinal herb needs to be processed in order to improve the efficacy and reduce the toxicity to humans. The medicinal properties of some herb extracts also could be changed to meet the diverse therapeutics acquirements. In China, it is well known that the processing technologies are the characteristic of TCM, and many herbs must be processed before they can be used in clinical prescription.
Gardeniae Fructus (Fruits of Gardenia jasminoides Ellis, Chinese name "Zhizi," GF), a frequently used TCM herb, has been broadly applied in clinical use and food additives in China and other Asian countries. GF extract has cold nature and bitter taste, the function of dispelling hot and eliminating dampness, cooling blood, and healing poison, alleviating pain according to the TCM theory [1]. GF extract could be used to treat various diseases, such as febrile diseases, jaundice, acute conjunctivitis, haematuria, epistaxis, and pyogenic infections [2-5]. The extract (Gardenia yellow) was widely used as a colorant in food additives [6]. 
GF could be processed into many different types of products including crude GF (CGF), stir-frying GF (SGF), gancao mix-frying GF (GCGF), ginger mix-frying GF (GIGF), charred GF, and carbonized GF in clinical application [7]. These types of GF extract have usually a common function while each product possesses its individual efficacy characteristics. For example, CGF and SGF extract could be used to dispel hot and GIGF extract could be used to stop vomiting. Meanwhile, the charred GF extract could be used to cool blood and carbonized GF extract could be used to stop bleeding [8].

Lots of studies have being focused on the chemical ingredients in GF. Some GF ingredients have been isolated and identified such as geniposides, geniposidic acid, genipin-1$\beta$-gentiobioside, gardenoside, shanzhiside, scandoside methyl ester, methyl deacetyl, deacetyl-asperulosidic acid methyl ester, crocin I, and crocin II $[9,10]$. It was reported that iridoids have antidepression $[11,12]$, antimicrobial activity [13], anti-inflammatory [14,15], and antitumor [16] effects, while crocins process the hepatoprotection [17], antihyperlipidemic [18], and antidiabetics [19], besides antidepression [20,21], anti-inflammatory [22], and antitumor [19] effects. Pharmacokinetics is an indispensable strategy to know drug behaviors in vivo after administration. Pharmacokinetic profiles of multiple components are beneficial for evaluating the safety and efficacy of herbal medicines [23]. Up to now, the pharmacokinetics of some active compounds in GF extract were studied by HPLC-UV [24,25] and HPLC-MS [26-28] analytical method. However, those pharmacokinetics studies of GF extract mainly focus on iridoid glycosides in vivo. To the best of our knowledge, there were no reports on the simultaneous pharmacokinetics of iridoids and crocin. In addition, there are no reports about the differences in pharmacokinetic parameters of bioactive compounds between after oral administration of the crude GFs and after other different processed GF extract.

In the present study, a rapid and sensitive HPLC-MS method was developed and validated for simultaneous determination of iridoids and crocins in rat plasma after oral administration of the crude and three processed GF aqueous extracts. The purpose of this study is to clarify the differences in pharmacokinetic parameters of bioactive compounds between after oral administration of the crude GF and the processed GF extract. It could also exhibit how processing method changed the pharmacokinetic action of bioactive components in vivo.

\section{Materials and Methods}

2.1. Materials and Reagents. The herb CGF and gancao (Glycyrrhizae Radix et Rhizoma) were obtained from Anguo TCM market (Hebei, China) and were authenticated by Professor Lin Ma (Tianjin University of Traditional Chinese Medicine). The voucher specimens were deposited at the lab of Tianjin University of Traditional Chinese Medicine. Fresh ginger was purchased from Tianjin market. Geniposidic acid, geniposide, genipin-1- $\beta$-gentiobioside, genipin, crocin I, crocin II, crocetin, and loganin (internal standard; IS) were purchased from the National Institute for the Control of
Pharmaceutical and Biological Products (Beijing, China). The purity of all standards was all above $98 \%$.

Methanol, acetonitrile (Merck, Darmstadt, Germany), and formic acid (Tedia Company Inc., Fairfield, USA) were of chromatographic grade. Purified water produced by a Milli-Q Academic ultra-pure water system (Millipore, Milford, MA, USA) was used in all experiments. All other reagents of analytical grade were obtained from Tianjin Concord Science Co. Ltd. (Tianjin, China).

2.2. Preparation of Gardeniae Fructus. The processing of SGF extract was that CGF material was tossed into a heated pan and stir-fryed until the surface of the GF slightly turns yellow or gives off an aroma. GCGF is stir-frying GF with gancao (Glycyrrhizae Radix et Rhizoma) extract solution (70 g gancao extracted with $168 \mathrm{~mL}$ water) that soaks into them. GIGF is stir-frying GF with ginger juice (12.5\% ginger juice) that soaks into the herb. $600 \mathrm{~g}$ CGF, SGF, GCGF, and GIGF were refluxed twice with water $(1: 10, \mathrm{w} / \mathrm{v})$ for $2 \mathrm{~h}$, respectively. The extraction was combined and condensed to $500 \mathrm{~mL}$. The concentrations of the residues were $1.2 \mathrm{~g} / \mathrm{mL}$ for CGF, SGF, GCGF, and GIGF extract.

\subsection{Quantitative Determination of Extracts by UHPLC-DAD.} Six analytes (geniposidic acid, geniposide, genipin-1$\beta$-gentiobioside, crocin I, crocin II, and crocetin) in GF extracts were quantitatively analyzed. Two grams of sample power (CGF, SGF, GCGF, and GIGF) was extracted twice with water $(1: 10, \mathrm{w} / \mathrm{v})$ under reflux in a water bath for $2 \mathrm{~h}$, respectively. After the extracts were filtered and combined, the solution was evaporated to dryness. The yields of CGF, SGF, GCGF, and GIGF extract were 32.99\%, 35.82\%, 35.8\%, and $36.13 \%$, respectively.

The extract $(20 \mathrm{mg})$ was precisely weighted and dissolved with methanol $(10 \mathrm{~mL})$ under ultrasonically for $30 \mathrm{~min}$. After replenished with methanol by weight loss, the extraction solution was diluted twice and filtered through a 0.22 membrane filter. The analyses were performed by UPLCPDA method according to our previous publication [29].

2.4. Quantitative Determination of Plasma Sample by LC-ESI$M S / M S$. The LC-ESI-MS/MS system is composed of an Agilent 1200 series LC system (Agilent Technologies, USA) including a binary pump, a vacuum degasser unit, an autosampler, and an API 3200 triple quadrupole mass Spectrometer with an ESI source (Concord, Ontario, Canada). Data was acquired by Analyst 1.4.2 software (AB MDS Sciex).

The Agilent Eclipse plus $\mathrm{C}_{18}(4.6 \times 100 \mathrm{~mm}, 1.8 \mu \mathrm{m})$ column with a guard column was used to separate multiple ingredients. The mobile phases were methanol (A) and water (B) with a gradient elution of $3-36 \% \mathrm{~A}$ at $0-3 \mathrm{~min}, 36-36 \% \mathrm{~A}$ at $3-15 \mathrm{~min}, 36-97 \% \mathrm{~A}$ at $15-16 \mathrm{~min}$, and $97-97 \% \mathrm{~A}$ at 16-26 min, then back from $97 \%$ to $3 \%$ balanced for $10 \mathrm{~min}$. The flow rate was set at $0.3 \mathrm{~mL} / \mathrm{min}$ and the column temperature was set at $30^{\circ} \mathrm{C}$. The injection volume was $5 \mu \mathrm{L}$. 
The detection was operated in Multiple Reaction Monitoring (MRM) mode. The source parameters were as follows: curtain gas, collision gas, ion spray voltage, temperature, ion source gas 1 , and ion source gas 2 were set at $20 \mathrm{psi}, 5 \mathrm{psi}, 5500 \mathrm{~V}, 450^{\circ} \mathrm{C}, 40 \mathrm{psi}$, and $40 \mathrm{psi}$, respectively. Nitrogen was the only gas used in the experiment. The other parameters are listed in Table 1.

\subsection{Preparation Standard Solution and Quality Control} Samples. The appropriate amounts of geniposidic acid, genipin-1- $\beta$-gentiobioside, geniposide, genipin, and crocetin were separately weighted and prepared in methanol as stock solutions. The IS stock solution of loganin was also prepared in methanol and kept at $100 \mathrm{ng} \cdot \mathrm{mL}^{-1}$ level in each working solution and sample. The stock solutions were prepared together as a mixed standard solution, and then it was gradually diluted into a series of concentrations as mixed working solution. All the working solutions were stored at $4^{\circ} \mathrm{C}$ before use.

Quality control (QC) samples containing geniposidic acid, geniposide, genipin-1- $\beta$-gentiobioside, genipin, and crocetin were prepared at LLOQ $(10 \mathrm{ng} / \mathrm{mL})$, low $(30 \mathrm{ng} /$ $\mathrm{mL})$, medium $(300 \mathrm{ng} / \mathrm{mL})$, and high concentrations $(3000 \mathrm{ng} / \mathrm{mL})$, spiking with appropriate standard solutions with blank plasma to establish calibration curve and method validation. The samples were prepared by the same procedures for plasma samples as described below.

2.6. Animal Experiment and Plasma Sample Preparation. A total of 24 male Sprague-Dawley rats (weight: 220-250 g) were obtained from animal center of Tianjin University of Traditional Chinese Medicine (Tianjin, China). Those rats were randomly divided into four groups (CGF, SGF, GCGF, and GIGF groups) and were housed in an environmentally controlled room (temperature $20-25^{\circ} \mathrm{C}$, humidity $60 \pm 5 \%$ ). The rats were allowed free accessibility to food and water in the first week. They were fasted for $12 \mathrm{~h}$ before the experiment with water taken freely. The animal experiments were according to the Guidelines for the Care and Use of Laboratory Animals and were approved by the Animal Ethics Committee of Tianjin University of Traditional Chinese Medicine. The rats were randomly divided into four groups. Six rats in each group were separately given single dose of $0.75 \mathrm{~mL} / \mathrm{kg}$ (0.9 g.kg herbal medicine) extract solution of four kinds of GFs vial oral administration. Blood samples (about $300 \mu \mathrm{L}$ ) were collected at $0,0.08,0.25,0.5,1,2,4,6,8$, $10,12,14$, and $24 \mathrm{~h}$. The blood samples were collected in heparinized tubes and immediately centrifuged at $6000 \mathrm{rpm}$ for $10 \mathrm{~min}$ at $4^{\circ} \mathrm{C}$. The plasma samples were stored at $-80^{\circ} \mathrm{C}$ until analysis.

The plasma sample $(100 \mu \mathrm{L})$ was spiked with $20 \mu \mathrm{L}$ IS working solution and $10 \mu \mathrm{L}$ formic acid in an Eppendorf tube. Samples were vortex for $30 \mathrm{~s}$, and then $1 \mathrm{~mL}$ acetonitrile was added to precipitate protein. Then samples were vortexed for $2 \mathrm{~min}$ and the tubes were centrifuged at $14000 \mathrm{rpm}$ for $10 \mathrm{~min}$. The upper organic phase was transferred into a new tube and evaporated to dryness under a flow of nitrogen gas. The residue was reconstituted with
TABLE 1: MRM scanning and mass spectrometry parameters of analytes and IS.

\begin{tabular}{lccccccc}
\hline \multirow{2}{*}{$\begin{array}{c}\text { Compounds } \\
\end{array}$} & Q1 & Q3 & DP & EP & CE & CXP & Rt.(min) \\
\hline Geniposidic acid & 373.0 & 123.2 & -35 & -6 & -27 & -2 & 9.33 \\
Genipin-1- & 548.9 & 224.8 & -57 & -7 & -22 & -5 & 12.29 \\
$\beta$-gentiobioside & & & & & & & \\
Geniposide & 386.8 & 224.9 & -37 & -3 & -15 & -2 & 14.72 \\
Genipin & 224.7 & 101.1 & -54 & -3 & -17 & -4 & 19.53 \\
Crocetin & 327.0 & 283.0 & -28 & -7 & -18 & -2 & 24.64 \\
Loganin (IS) & 389.2 & 227.1 & -26 & -3 & -15 & -4 & 15.8 \\
\hline
\end{tabular}

$50 \%$ methanol $100 \mu \mathrm{L}$. After centrifuging at $14000 \mathrm{rpm}$ for $10 \mathrm{~min}$, the supernatant was transferred into an autosampler vial and a volume of $5 \mu \mathrm{L}$ was injected into the HPLC-ESIMS/MS for analysis.

2.7. Method Validation. The method was validated in terms of specificity, linearity, accuracy and precision, recovery, matrix effect, and stability according to the USA Food and Drug Administration (FDA) bioanalytical method validation guidelines.

2.7.1. Method Validation Linearity. The calibration curves were constructed to calculate the linearity by the plot of the peak area ratios of analytes versus the IS against the concentrations using a $1 / X^{2}$ weighted linear least-squares regression model. The lower limit of quantification (LLOQ) of the assay was defined as the lowest concentration of the standard curve at which the signal-to-noise ratio $(S / N)$ was preliminary found to be larger than 5. It was acceptable that the precision was less than $20 \%$ and the accuracy was within $\pm 20 \%$.

2.7.2. Specificity. Blank plasma samples were obtained from six individual rats. The blank plasma, blank plasma spiked with mixed standard solution, and the real plasma samples were compared by their chromatographic profiles to exclude endogenous interference.

2.7.3. Precision and Accuracy. The precision and accuracy were assessed by analyzing QC samples at LLOQ, low, medium, and high concentrations. The intraday precision and accuracy were evaluated by six-replicate quality control samples in the same day. The interday precision and accuracy were evaluated by six-replicate samples on three consecutive days. The precision was expressed by the relative standard deviation (RSD).

2.7.4. Extraction Recovery and Matrix Effect. The extraction recoveries of 5 ingredients from GF were determined at four QC levels with six replicates. It was calculated by comparing the peak areas ratios of the processed samples with those of postprocessed spiked samples. The matrix effects were calculated by comparing the peak areas ratios of the analytes in 
postprocessed spiked samples with those of the analytes in pure standard solution.

2.7.5. Stability. The stability of 5 components in the plasma was obtained by evaluating QC samples at four concentration levels with three replicates in different conditions. The QC samples were kept at room temperature for $12 \mathrm{~h}$ to determine the short-term stability. Freeze-thaws stability was tested after three freeze-thaw $\left(-80^{\circ} \mathrm{C}\right.$ at room temperature) on three consecutive days. Long-term stability was tested by evaluating QC samples stored at $-80^{\circ} \mathrm{C}$ for one month.

2.8. Pharmacokinetics Study and Statistical Analysis. The pharmacokinetic parameters were calculated using a Drug and Statistics 1.0 (DAS 1.0) software (Medical College of Wannan, China). The pharmacokinetic parameters included time of maximum concentration $\left(T_{\max }\right)$, maximum plasma concentration $\left(C_{\max }\right)$, elimination half-life $\left(T_{1 / 2}\right)$, and area under concentrations curve $\left(\mathrm{AUC}_{0-\mathrm{t}}\right.$ and $\left.\mathrm{AUC}_{0-\infty}\right)$. All values were expressed as mean $\pm \mathrm{SD}$. A paired $t$ test was used to test the difference among these groups.

\section{Results and Discussion}

3.1. The Content of the Six Analytes in GFs Extracts. The original content of compounds in herb extract was a nonnegligible factor to influence the pharmacokinetics results. The contents of geniposidic acid, genipin-1- $\beta$-gentiobioside, geniposide, crocin I, crocin II, and crocetin are $0.449,1.48$, 5.374, 0.327, 0.058, and 0.014\% in CGF extract; 0.429, 1.574, $5.079,0.403,0.066$, and $0.016 \%$ in SGF extract; 0.421, 1.488, $5.058,0.384,0.064$, and $0.017 \%$ in GCGF extract; 0.414 , $1.598,5.241,0.377,0.066$, and $0.015 \%$ in GIGF extract, respectively. Compared with CGF extract, the contents of geniposidic acid and geniposide in the three processing products were reduced. On the contrary, the contents of genipin-1- $\beta$-gentiobioside were increased in processed ones with different processed method. For all three crocin compounds, the contents of crocin I, crocin II, and crocetin in the three processed GF extracts were all higher than those in CGF. These results would contribute to the analysis of the exposure level in blood and make the pharmacokinetics study more objective and reasonable.

3.2. Optimization of Method Condition. To achieve better resolution and good peak shape, the chromatographic conditions were optimized by using methanol, acetonitrile, water, and water with different proportions of formic acid. As a result, acetonitrile-water was chosen as the mobile phase to obtain high response intensity and good peak shape for the five analytes. Both positive and negative detection modes were compared to get better mass response of analytes. Negative ionization mode was chosen and MRM mode was applied due to its higher sensitivity. The related mass parameters of all five analytes and IS were optimized to obtain better ionization efficiency (Table 1).
Liquid-liquid extraction (LLE) and protein precipitation (PPT) methods were examined to extract the iridoids and crocins from plasma samples. Ethyl acetate containing different concentrations of formic acid was estimated as LLE solvents. Methanol, acetonitrile, and acetonitrile added different concentrations of formic acid were tested for PPT. It was found that acetonitrile and formic acid were optimized as the pretreatment method of plasma samples according to negligible matrix effect and high extraction recovery for all analytes.

\subsection{Method Validation}

3.3.1. Specificity. The specificity of LC-MS/MS was evaluated by analyzing blank plasma samples, blank plasma spiked with mixed standards, and plasma obtained after oral administration for $30 \mathrm{~min}$ of GF. As shown in Figure 1, there were no interferences and endogenous interference at their peak region in the chromatogram profile.

3.3.2. Linearity and $L L O Q$. The linear range was from 10 to $3000 \mathrm{ng} / \mathrm{mL}$ for the five components. Good linearity was determined in the validation concentration range (all correlation coefficients $>0.994)$. The calibration curve equations and correlation coefficients of the ingredients were as follows: $y=0.00328 x+0.0115, \quad r=0.9944$ for geniposidic acid, $y=0.00629 x+0.00621, \quad r=0.9976$ for genipin- $1-\beta$ gentiobioside, $y=0.00252 x+0.00791, r=0.9961$ for geniposide, $y=0.00115 x+0.00438, r=0.9985$ for genipin, and $y=0.00341 x+0.0397, r=0.9952$ for crocetin. The lower limits of quantification (LLOQ) for determination of all five analytes were all $10 \mathrm{ng} / \mathrm{mL}$. These results illustrated that the newly LC-MS/MS method was proper for the quantitative detection.

3.3.3. Accuracy and Precision. The intraday and interday precision and accuracy were determined by replicate analysis of QC samples on the same day (intraday) and continuously for 3 days (interday), respectively. The intraday and interday precision and accuracy are shown in Table 2. The intraday and interday precisions of the analytes met the requirement of method validation, and the accuracies were in the range of $91 \%-105 \%$. The results demonstrated that the precision and accuracy of the newly LC-MS/MS method were accurate, reliable, reproducible, and acceptable.

3.3.4. Extraction Recovery and Matrix Effect. The mean extraction recoveries of 5 analytes at four different concentrations were ranged from $81.0 \pm 7.4 \%$ to $114 \pm 5 \%$ with RSD less than $13.55 \%$. The matrix effect was from $88.6 \pm 5.7 \%$ to $115 \pm 2 \%$ with RSD less than $13.47 \%$. The data showed that the extraction recovery and matrix effect of this method were reliable and reproducible. The extraction efficiencies of 5 ingredients were acceptable (Table 3).

3.3.5. Stability. The stability of the five components in rat plasma was determined by evaluating QC samples stored at 

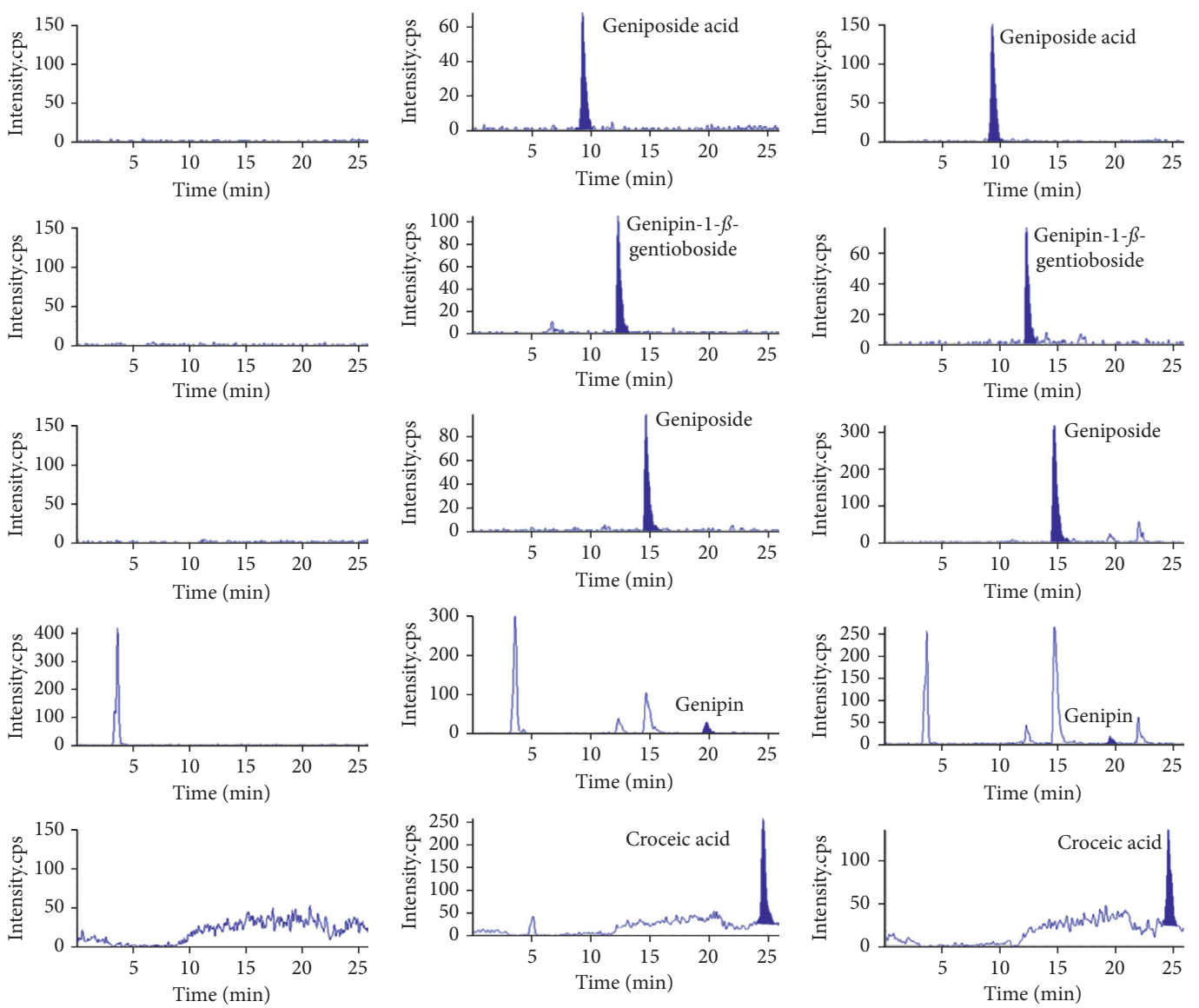

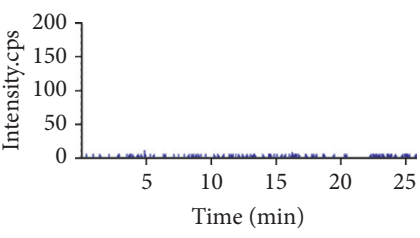

(a)

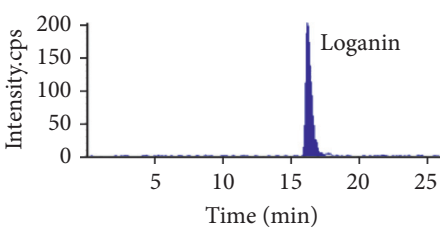

(b)

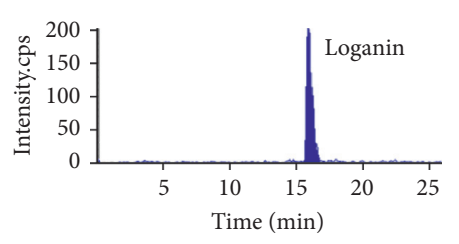

(c)

FIGURE 1: MRM chromatograms of all components in rat plasma: (a) blank plasma; (b) blank plasma spiked with five analytes at LLOQ $(10 \mathrm{ng} / \mathrm{mL})$ and IS; (c) the plasma sample at $0.5 \mathrm{~h}$ after oral administration of CGF.

different temperature and timing conditions. All the components were stable in the autosampler for $24 \mathrm{~h}$, after three freeze-thaw cycles and at a month at $-80^{\circ} \mathrm{C}$. As listed in Table 4, the results indicated that the five analytes in rat plasma were stable at different storage conditions with an RSD range of $1.03-13.44 \%$.

3.4. Pharmacokinetics Study. The newly established LCMS/MS method was validated and applied to pharmacokinetics studies of five bioactive components in the plasma after oral administration of CGF, SGF, GCGF, and GIGF aqueous extracts to rats. Five components were distributed as an opened single-compartment model, and the mean pharmacokinetic profiles are presented in Figure 2. Meanwhile, the pharmacokinetic parameters including $\mathrm{AUC}_{(0-\mathrm{t} 24)}, \mathrm{AUC}_{(0-\infty)}, C_{\max }, T_{1 / 2}$, and $T_{\max }$ are summarized in Table 5.
After oral administration of CGF extract, $T_{\max }$ was ranged from 0.55 to $3.33 \mathrm{~h}$ and $T_{1 / 2}$ was ranged from 0.72 to $3.42 \mathrm{~h}$ for four iridoids while $T_{\max }$ was $7.30 \pm 3.93 \mathrm{~h}$ and $T_{1 / 2}$ was $41.65 \pm 57.62 \mathrm{~h}$ for crocetin. It was indicated that iridoids exhibited more rapid absorption than crocins in GF extract. Geniposidic acid, which had lower content than genipin-1$\beta$-gentiobioside and geniposide, presented a higher exposure level. The values of $\mathrm{AUC}_{(0-24 \mathrm{~h})}$ and $\mathrm{AUC}_{(0-\infty)}$ of geniposidic acid were obviously higher than those of other compounds. Geniposide, which was the highest content in GF extracts, showed a lower exposure level than geniposidic acid. Genipin could be hardly determined in GF extracts, but it could be detected in plasma samples after oral administration of GF extract. This phenomenon might be related to the fact that geniposide was hydrolyzed into genipin with the help of intestinal flora [24]. Crocin I and crocin II were determined in GF extract, but they could not be determined in rat plasma after oral administration of GF extract. 
TABLE 2: Intraday and interday precision and accuracy of 5 compounds $(n=6)$.

\begin{tabular}{|c|c|c|c|c|c|}
\hline \multirow{2}{*}{ Compounds } & \multirow{2}{*}{ Concentration (ng/mL) } & \multicolumn{2}{|c|}{ Intraday } & \multicolumn{2}{|c|}{ Interday } \\
\hline & & RSD (\%) & Accuracy (\%) & RSD (\%) & Accuracy (\%) \\
\hline \multirow{4}{*}{ Geniposidic acid } & 10 & 4.94 & 104 & 2.62 & 101 \\
\hline & 30 & 3.83 & 99.5 & 0.55 & 99.6 \\
\hline & 300 & 5.55 & 105 & 3.19 & 101 \\
\hline & 3000 & 3.58 & 100 & 2.61 & 97 \\
\hline \multirow{4}{*}{ Genipin-1- $\beta$-gentiobioside } & 10 & 5.29 & 102 & 0.79 & 101 \\
\hline & 30 & 3.63 & 99 & 5.20 & 99 \\
\hline & 300 & 4.50 & 93 & 4.10 & 98.9 \\
\hline & 3000 & 2.04 & 98 & 3.03 & 98.2 \\
\hline \multirow{4}{*}{ Geniposide } & 10 & 3.66 & 96.5 & 4.51 & 97.7 \\
\hline & 30 & 4.67 & 98 & 3.54 & 95 \\
\hline & 300 & 5.03 & 94 & 3.15 & 96.6 \\
\hline & 3000 & 3.68 & 93 & 0.56 & 93.9 \\
\hline \multirow{4}{*}{ Genipin } & 10 & 7.35 & 96.5 & 2.20 & 98.7 \\
\hline & 30 & 9.32 & 95 & 3.19 & 99 \\
\hline & 300 & 3.61 & 91 & 1.71 & 92.9 \\
\hline & 3000 & 4.14 & 94.5 & 2.43 & 91.3 \\
\hline \multirow{4}{*}{ Crocetin } & 10 & 8.84 & 100 & 2.08 & 97.4 \\
\hline & 30 & 4.71 & 98 & 0.56 & 98.6 \\
\hline & 300 & 3.66 & 97.5 & 1.32 & 96.7 \\
\hline & 3000 & 8.37 & 96 & 1.14 & 97.1 \\
\hline
\end{tabular}

TABle 3: Recoveries and matrix effects of 5 compounds $(n=3)$.

\begin{tabular}{|c|c|c|c|c|c|}
\hline \multirow{2}{*}{ Compounds } & \multirow{2}{*}{ Concentration $(\mathrm{ng} / \mathrm{mL})$} & \multicolumn{2}{|c|}{ Recovery } & \multicolumn{2}{|c|}{ Matrix effect } \\
\hline & & Mean \pm SD (\%) & RSD (\%) & Mean \pm SD (\%) & RSD (\%) \\
\hline \multirow{4}{*}{ Geniposidic acid } & 10 & $96.7 \pm 4.8$ & 4.96 & $114 \pm 10$ & 8.77 \\
\hline & 30 & $110 \pm 13$ & 11.82 & $103 \pm 10$ & 9.71 \\
\hline & 300 & $111 \pm 6$ & 5.41 & $110 \pm 4$ & 3.64 \\
\hline & 3000 & $97.8 \pm 4.1$ & 4.19 & $115 \pm 2$ & 1.74 \\
\hline \multirow{4}{*}{ Genipin-1- $\beta$-gentiobioside } & 10 & $97.2 \pm 5.5$ & 5.66 & $92.5 \pm 7.5$ & 8.11 \\
\hline & 30 & $94.7 \pm 2.1$ & 2.22 & $114 \pm 4$ & 3.51 \\
\hline & 300 & $100 \pm 12$ & 12.01 & $88.6 \pm 5.7$ & 6.43 \\
\hline & 3000 & $94.8 \pm 4.1$ & 4.32 & $93.9 \pm 4.7$ & 5.01 \\
\hline \multirow{4}{*}{ Geniposide } & 10 & $97.4 \pm 9.4$ & 9.65 & $97.3 \pm 11.5$ & 11.82 \\
\hline & 30 & $110 \pm 4$ & 3.64 & $96.4 \pm 3.7$ & 3.84 \\
\hline & 300 & $84.3 \pm 4.0$ & 4.74 & $106 \pm 6.6$ & 6.23 \\
\hline & 3000 & $104 \pm 3$ & 2.88 & $111 \pm 6$ & 5.41 \\
\hline \multirow{4}{*}{ Genipin } & 10 & $81.2 \pm 11.0$ & 13.55 & $106 \pm 9$ & 8.49 \\
\hline & 30 & $81.0 \pm 7.4$ & 9.14 & $115 \pm 2$ & 1.74 \\
\hline & 300 & $111 \pm 11$ & 9.91 & $104 \pm 9$ & 8.65 \\
\hline & 3000 & $114 \pm 5$ & 4.39 & $92.9 \pm 5.2$ & 5.60 \\
\hline \multirow{4}{*}{ Crocetin } & 10 & $103 \pm 8$ & 7.77 & $89.1 \pm 4.2$ & 4.71 \\
\hline & 30 & $95.8 \pm 10.0$ & 10.44 & $94.3 \pm 6.8$ & 7.21 \\
\hline & 300 & $99.2 \pm 13.4$ & 13.51 & $98.0 \pm 13.2$ & 13.47 \\
\hline & 3000 & $98.5 \pm 10.0$ & 10.15 & $98.3 \pm 5.4$ & 5.49 \\
\hline
\end{tabular}

Crocetin, whose content was lower than those of crocin I and crocin II in GF extract, showed a relatively higher blood exposure level. The reason may be that crocin was transformed into crocetin quickly in the gastrointestinal tract after oral administration of crocin, and the exposure of its metabolite, crocetin, was much higher than crocin [30].

Regarding CGF extract as a control group, $C_{\max }$ (ng.mL $\left.{ }^{-1}\right), \mathrm{AUC}_{(0-24 \mathrm{~h})}(\mathrm{ng} / \mathrm{L} \cdot \mathrm{h})$, and $\mathrm{AUC}_{(0-\infty)}(\mathrm{ng} / \mathrm{L} \cdot \mathrm{h})$ of geniposidic acid and geniposide of SGF-, GCGF and GIGF extract groups were significant $(p<0.05)$. The values of these three parameters of geniposidic acid and geniposide in plasma after intragastric administration of three processed extracts were significantly decreased. Those three parameters of genipin-1- $\beta$-gentiobioside, genipin, and crocetin of SGFand GCGF-treated group were not significantly different. $T_{\max }$ of genipin-1- $\beta$-gentiobioside and crocetin were significantly different. Setting SGF extract treated group as a control group, $C_{\max }$ of geniposide was significantly 
TABLE 4: Stability of the 5 compounds $(n=6)$.

\begin{tabular}{|c|c|c|c|c|c|c|c|}
\hline \multirow{2}{*}{ Compounds } & \multirow{2}{*}{ Concentration $(\mathrm{ng} / \mathrm{mL})$} & \multicolumn{2}{|c|}{ Freeze-thaw cycles } & \multicolumn{2}{|c|}{ At $-80^{\circ} \mathrm{C}$ for a month } & \multicolumn{2}{|c|}{ Autosampler for $24 \mathrm{~h}$} \\
\hline & & RSD (\%) & Remain (\%) & RSD (\%) & Remain (\%) & RSD (\%) & Remain (\%) \\
\hline \multirow{4}{*}{ Geniposidic acid } & 10 & 10.37 & 96.3 & 7.48 & 107 & 1.42 & 94.7 \\
\hline & 30 & 5.71 & 101 & 10.14 & 98.0 & 6.05 & 96.4 \\
\hline & 300 & 6.44 & 107 & 5.30 & 106 & 3.40 & 96.4 \\
\hline & 3000 & 2.43 & 108 & 1.55 & 99.9 & 3.94 & 96.7 \\
\hline \multirow{4}{*}{ Genipin-1- $\beta$-gentiobioside } & 10 & 3.79 & 104 & 13.44 & 95.8 & 10.40 & 99.3 \\
\hline & 30 & 1.03 & 105 & 10.50 & 96.3 & 1.52 & 100 \\
\hline & 300 & 7.71 & 101 & 4.19 & 95.1 & 5.96 & 101 \\
\hline & 3000 & 2.60 & 103 & 0.87 & 95.1 & 2.75 & 90.7 \\
\hline \multirow{4}{*}{ Geniposide } & 10 & 4.10 & 97.5 & 10.75 & 99.2 & 10.57 & 102 \\
\hline & 30 & 5.17 & 92.8 & 9.85 & 98.4 & 4.11 & 95.1 \\
\hline & 300 & 1.88 & 93.1 & 2.79 & 94.8 & 7.91 & 96.3 \\
\hline & 3000 & 5.92 & 95.0 & 2.97 & 92.8 & 1.24 & 91.6 \\
\hline \multirow{4}{*}{ Genipin } & 10 & 3.81 & 101. & 3.14 & 108 & 3.24 & 95.4 \\
\hline & 30 & 4.43 & 95.3 & 12.68 & 103 & 4.49 & 104 \\
\hline & 300 & 2.68 & 95.9 & 5.98 & 105 & 0.76 & 90.6 \\
\hline & 3000 & 9.22 & 100 & 6.99 & 101 & 4.12 & 92.6 \\
\hline \multirow{4}{*}{ Crocetin } & 10 & 2.02 & 102 & 9.75 & 98.4 & 6.43 & 97.9 \\
\hline & 30 & 8.05 & 95.3 & 10.15 & 101 & 6.00 & 99.9 \\
\hline & 300 & 7.02 & 98.6 & 6.67 & 105 & 4.41 & 96.3 \\
\hline & 3000 & 6.52 & 106 & 1.33 & 103 & 5.55 & 98.9 \\
\hline
\end{tabular}

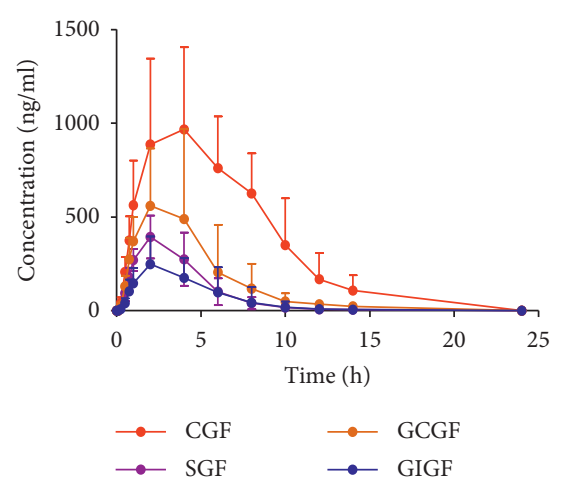

(a)

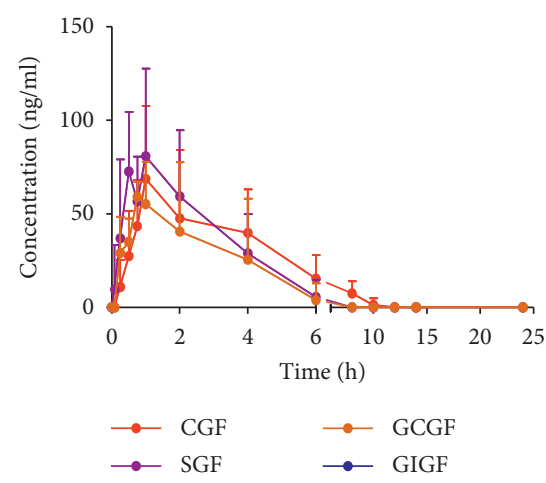

(d)

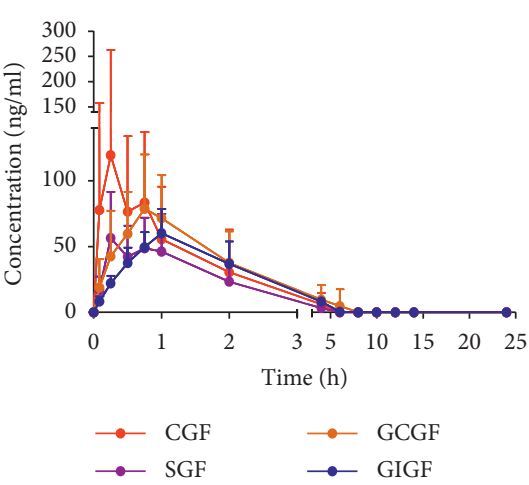

(b)

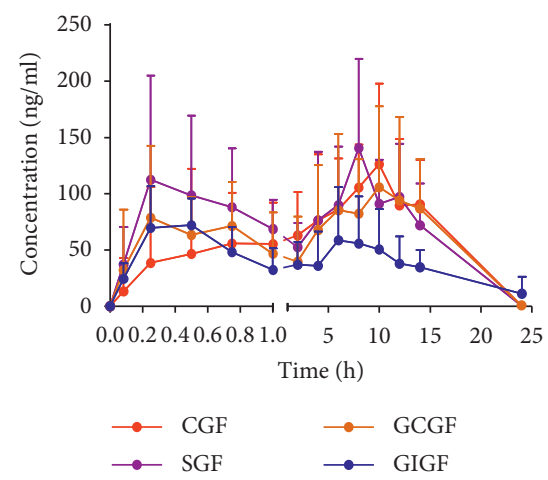

(e)

FIGURE 2: Means pharmacokinetic profiles multiple bioactive components after oral administration of CGF, SGF, GCGF, and GIGF extract to rats $(n=6)$. (a) Geniposidic acid, (b) genipin-1- $\beta$-gentiobioside, (c) geniposide, (d) genipin, and (e) crocetin.

decreased comparing with GCGF extract-treated and GIGF extract-treated groups. This result indicated that ginger mixfrying could reduce the bioavailability of geniposide. It was worth noticing that the absorption of geniposidic acid in rat plasma was decreased sharply after oral administration of processed GFs $\left(\mathrm{AUC}_{(0-\mathrm{t} 24)}\right.$, CGF : SGF: GCGF: GIGF, 1: 
TABLE 5: The main pharmacokinetics of 5 compounds in rat plasma $(n=6)$.

\begin{tabular}{|c|c|c|c|c|c|c|}
\hline Compounds & Treatment & $C_{\max }(\mathrm{ng} / \mathrm{mL})$ & $T_{\max }(\mathrm{h})$ & $T_{1 / 2}(\mathrm{~h})$ & $\operatorname{AUC}_{(0-24 \mathrm{~h})}(\mathrm{ng} / \mathrm{L} \cdot \mathrm{h})$ & $\mathrm{AUC}_{(0-\infty)}(\mathrm{ng} / \mathrm{L} \cdot \mathrm{h})$ \\
\hline \multirow{4}{*}{ Geniposidic acid } & CGF & $1022.83 \pm 450.17$ & $3.33 \pm 1.63$ & $2.52 \pm 0.64$ & $7696.99 \pm 2741.28$ & $8586.63 \pm 3450.88$ \\
\hline & SGF & $398.33 \pm 107.8^{* *}$ & $1.78 \pm 0.44^{*}$ & $2.93 \pm 3.45$ & $1730.86 \pm 618.74^{* *}$ & $1774.38 \pm 654.94^{* *}$ \\
\hline & GCGF & $669.63 \pm 431.89$ & $3.33 \pm 3.43$ & $1.72 \pm 0.69^{*}$ & $2988.13 \pm 2302.06^{* *}$ & $3250.09 \pm 2342^{* *}$ \\
\hline & GIGF & $272.00 \pm 164.04^{* *}$ & $2.40 \pm 1.26$ & $1.50 \pm 0.42^{* *}$ & $1187.21 \pm 813.41^{* *}$ & $1351.01 \pm 827.12^{* *}$ \\
\hline \multirow{4}{*}{ Genipin-1- $\beta$-gentiobioside } & CGF & $149.90 \pm 132.07$ & $0.55 \pm 0.33$ & $0.72 \pm 0.25$ & $141.12 \pm 104.11$ & $237.29 \pm 119.51$ \\
\hline & SGF & $79.08 \pm 39.47$ & $0.61 \pm 0.31$ & $0.61 \pm 0.18$ & $109.26 \pm 58.78$ & $121.03 \pm 54.86^{*}$ \\
\hline & GCGF & $102.92 \pm 58.15$ & $0.78 \pm 0.20$ & $0.78 \pm 0.32$ & $206.27 \pm 142.55$ & $233.30 \pm 165.74$ \\
\hline & GIGF & $62.94 \pm 16.81$ & $1.05 \pm 0.37^{* \#}$ & $0.91 \pm 0.36^{\#}$ & $131.30 \pm 36.78$ & $151.67 \pm 48.73$ \\
\hline \multirow{4}{*}{ Geniposide } & CGF & $1071.67 \pm 598.46$ & $1.54 \pm 1.30$ & $3.21 \pm 2.31$ & $4000.13 \pm 1677.19$ & $4119.45 \pm 1649.61$ \\
\hline & SGF & $455.44 \pm 160.36^{*}$ & $0.89 \pm 0.72$ & $1.61 \pm 0.63$ & $1141.92 \pm 389.07^{* *}$ & $1200.78 \pm 382.09^{* *}$ \\
\hline & GCGF & $581.00 \pm 240.71^{*}$ & $0.86 \pm 0.13$ & $1.44 \pm 0.53^{*}$ & $1862.67 \pm 1078.69^{* *}$ & $1899.73 \pm 1092.04^{* *}$ \\
\hline & GIGF & $211.50 \pm 109.10^{* * \# \#}$ & $1.30 \pm 0.48$ & $1.08 \pm 0.45^{*}$ & $733.81 \pm 519.14^{* *}$ & $781.95 \pm 530.75^{* *}$ \\
\hline \multirow{4}{*}{ Genipin } & CGF & $76.44 \pm 40.93$ & $1.11 \pm 0.40$ & $3.42 \pm 1.94$ & $265.30 \pm 103.43$ & $356.72 \pm 139.94$ \\
\hline & SGF & $98.05 \pm 41.84$ & $1.00 \pm 0.55$ & $1.39 \pm 0.88$ & $233.77 \pm 141.78$ & $281.41 \pm 178.60$ \\
\hline & GCGF & $68.52 \pm 19.41$ & $1.10 \pm 0.52$ & $1.39 \pm 0.98$ & $152.67 \pm 113.60$ & $329.06 \pm 394.68$ \\
\hline & GIGF & - & - & - & - & - \\
\hline \multirow{4}{*}{ Crocetin } & CGF & $165.02 \pm 52.86$ & $7.30 \pm 3.93$ & $41.65 \pm 57.62$ & $1251.80 \pm 354.98$ & $10824.60 \pm 13505.86$ \\
\hline & SGF & $152.51 \pm 81.90$ & $6.61 \pm 4.05$ & $56.17 \pm 42.99$ & $1258.95 \pm 596.55$ & $7510.78 \pm 8494.79$ \\
\hline & GCGF & $162.66 \pm 76.43$ & $6.43 \pm 5.02$ & $12.75 \pm 6.64^{\#}$ & $1098.06 \pm 522.58$ & $3845.62 \pm 5252.68$ \\
\hline & GIGF & $91.18 \pm 35.65^{*}$ & $1.29 \pm 2.31^{* \#}$ & $31.84 \pm 29.27$ & $621.22 \pm 389.71^{* \#}$ & $3610.39 \pm 2206.09$ \\
\hline
\end{tabular}

CGF as control group, $p<0.05^{*} ; p<0.01^{* *}$, SGF as control group, $p<0.05^{\#} ; p<0.01^{\# \#}$.

$0.22: 0.39: 0.15)$, while the content of geniposidic acid in herbal extracts declined very slightly after processing (content, CGF : SGF : GCGF : GIGF, $1: 0.94: 0.74: 0.76$ ). It was indicated that the processing method could reduce the absorption of geniposidic acid in vivo. $T_{\max }$ and $\mathrm{AUC}_{(0-24 \mathrm{~h})}$ parameters of crocetin in GIGF extract were significantly different $(p<0.05)$.

Genipin could be hardly determined in GF extract and it could be detected in plasma after oral CGF, SGF, and GCGF extract as a metabolite of geniposide. Genipin could not be detected in GIGF extract, which was probably caused by the inhibition of absorption after ginger mix-frying. Interestingly, as for genipin-1- $\beta$-gentiobioside, an additional small peak was observed before the maximum plasma concentration in CGFand SGF-treated groups, and one peak only appeared in GCGFand GIGF-treated groups (Figure 2(b)). The same inconsistent phenomenon also emerged in crocetin as shown in Figure 2(e), double-peak phenomenon was found in SGF-, GCGF-, and GIGF-treated groups, while one-peak phenomenon was observed in CGF-treated group. Their $T_{\max }$ also occurred at different times; thus, it was at $1.29 \pm 2.31 \mathrm{~h}$ for the GIGF-treated group and 6.43-6.61 h for SGF- and GCGF-treated groups. The reasons for all those results need further detailed investigation.

\section{Conclusion}

A rapid, sensitive, and stable LC-MS/MS method was established for simultaneous quantification of geniposidic acid, geniposide, genipin-1- $\beta$-gentiobioside, genipin, and crocetin in rat plasma. The method validation was successfully been used in the pharmacokinetics study of five components after oral administration of the different GF extract. The results exhibited that different processing methods could obviously affect the absorption of geniposidic acid, geniposide, genipin-1- $\beta$-gentiobioside, genipin, and crocetin in rats. The differences of pharmacokinetic parameter were probably induced by the processing progress affecting the content and inhibiting the absorption of their two respects. In the future, the intensive study that processing inhibited absorption of geniposide and then changed the production of genipin is necessary, to prove the processing progress changed the physiological disposition and metabolic profile of the components. Further research on metabolic profile change of crocin I and crocin II is also needed.

\section{Data Availability}

The data used to support the findings of this study are included within the article.

\section{Conflicts of Interest}

The authors declare no conflicts of interest.

\section{Authors' Contributions}

Yan-xu Chang acquired funding for the research. Yan-xu Chang and Jin Li designed the work. Xi Yang performed the experiments. Xi Yang, Xuejing Yang, and Jin Li analyzed the data. Xi Yang, Jin Li, Xuejing Yang, and Yan-xu Chang wrote the manuscript. Jun $\mathrm{He}$ and Yan-xu Chang reviewed the manuscript. Jin Li contributed equally to this work. All authors discussed the results and approved the final manuscript.

\section{Acknowledgments}

This research was supported by National Natural Science Foundation of China (81973704, 81374050); Tianjin 
Research Program of Application Foundation and Advanced Technology (18JCYBJC95000); and Special Program of Talents Development for Excellent Youth Scholars in Tianjin.

\section{References}

[1] Chinese Pharmacopoeia Commission, Pharmacopoeia of the People's Republic of China, Chinese Pharmacopoeia Commission, Beijing, China, 2015.

[2] E.-H. Park, M.-H. Joo, S.-H. Kim, and C.-J. Lim, "Antiangiogenic activity of Gardenia jasminoides fruit," Phytotherapy Research, vol. 17, no. 8, pp. 961-962, 2003.

[3] B. Li, "A survey of the research on Fructus Gardenia," Lishizhen Medicine and Materia Medica Research, vol. 15, pp. 370-371, 2004.

[4] F. Yin and J.-H. Liu, "Research and application progress of Gardenia jasminoides," Chinese Herbal Medicines, vol. 10, no. 4, pp. 362-370, 2018.

[5] Q. C. Chen, W. Y. Zhang, H. J. Kim et al., "Effects of Gardeniae fructus extract and geniposide on promoting ligament cell proliferation and collagen synthesis," Phytotherapy Research, vol. 24, no. S1, pp. S1-S5, 2010.

[6] S. Yamada, H. Oshima, I. Saito, and J. Hayakawa, "Adoption of crocetin as an indicator compound for detection of gardenia yellow in food products," Food Hygiene and Safety Science, vol. 37, no. 6, pp. 372-377, 1996.

[7] T. Wu, L. Yan, and M. X. Zhu, "History and modern research of processed products of Gardenia jasminoides Ellis," China Pharmacist, vol. 18, pp. 1011-1014, 2015.

[8] X. L. Zhang, H. L. Cheng, and H. F. Li, "History of processed products of Chardenia jasminoides Ellis," Chinese Traditional Patent Medicine, vol. 27, pp. 1281-1283, 2005.

[9] Y. Han, J. Wen, T. T. Zhou, and G. R. Fan, "Chemical fingerprinting of Gardenia jasminoides Ellis by HPLC-DADESI-MS combined with chemometrics methods," Food Chemistry, vol. 188, pp. 648-657, 2015.

[10] X. Y. Wu, Y. Zhou, F. Z. Yin et al., "Quality control and producing areas differentiation of gardeniae fructus for eight bioactive constituents by HPLC-DAD-ESI/MS," Phytomedicine, vol. 21, no. 4, pp. 551-559, 2014.

[11] J. S. Tian, Y. L. Cui, L. M. Hu et al., "Antidepressant-like effect of genipin in mice," Neuroscience Letters, vol. 479, no. 3, pp. 236-239, 2010.

[12] L. Cai, R. Li, W.-J. Tang, G. Meng, X.-Y. Hu, and T.-N. Wu, "Antidepressant-like effect of geniposide on chronic unpredictable mild stress-induced depressive rats by regulating the hypothalamus-pituitary-adrenal axis," European Neuropsychopharmacology, vol. 25, no. 8, pp. 1332-1341, 2015.

[13] I. Kyoko, Y. Masae, and T. Shuzo, "Studies on iridoid-related compounds. II. The structure and antimicrobial activity of aglucones of galioside and gardenoside," Journal of Natural Products, vol. 46, no. 4, pp. 532-536, 1983.

[14] H.-J. Koo, Y.-S. Song, H.-J. Kim et al., "Antiinflammatory effects of genipin, an active principle of gardenia," European Journal of Pharmacology, vol. 495, no. 2-3, pp. 201-208, 2004.

[15] H.-J. Koo, K.-H. Lim, H.-J. Jung, and E.-H. Park, “Anti-inflammatory evaluation of gardenia extract, geniposide and genipin," Journal of Ethnopharmacology, vol. 103, no. 3, pp. 496-500, 2006.

[16] M. K. Shanmugam, H. Y. Shen, F. R. Tang et al., "Potential role of genipin in cancer therapy," Pharmacological Research, vol. 133, pp. 195-200, 2018.
[17] T.-H. Tseng, C.-Y. Chu, J.-M. Huang, S.-J. Shiow, and C.-J. Wang, "Crocetin protects against oxidation damage in rat primary hepatocytes," Cancer Letters, vol. 97, no. 1, pp. 61-67, 1995.

[18] F. T. Tang, Z. Y. Qian, P. Q. Liu et al., "Crocetin improves endothelium-dependent relaxation of thoracic aorta in hypercholesterolemic rabbit by increasing eNOS activity," Biochemical Pharmacology, vol. 72, no. 5, pp. 558-565, 2006.

[19] M. Hashemi and H. Hosseinzadeh, "A comprehensive review on biological activities and toxicology of crocetin," Food and Chemical Toxicology, vol. 130, pp. 44-60, 2019.

[20] B. Amin, A. Nakhsaz, and H. Hosseinzadeh, "Evaluation of the antidepressant-like effects of acute and sub-acute administration of crocin and crocetin in mice," Avicenna Journal of Phytomedicine, vol. 5, no. 5, pp. 458-468, 2015.

[21] F. V. Hassani, V. Naseri, B. M. Razavi, S. Mehri, K. Abnous, and H. Hosseinzadel, "Antidepressant effects of crocin and its effects on transcript and protein levels of CREB, BDNF, and VGF in rat hippocampus," DARU Journal of Pharmaceutical Sciences, vol. 22, no. 1, pp. 16-24, 2014.

[22] Y. Y. Hu, X. Liu, Q. Xia et al., "Comparative anti-arthritic investigation of iridoid glycosides and crocetin derivatives from Gardenia jasminoides Ellis in Freund's complete adjuvant-induced arthritis in rats," Phytomedicine, vol. 53, pp. 223-233, 2019.

[23] D. K. Walker, "The use of pharmacokinetic and pharmacodynamic data in the assessment of drug safety in early drug development," British Journal of Clinical Pharmacology, vol. 58, no. 6, pp. 601-608, 2004.

[24] K. Ueno, Y. Takeda, Y. Iwasaki, and F. Yoshizaki, "Simultaneous estimation of geniposide and genipin in mouse plasma using high-performance liquid chromatography," Analytical Sciences, vol. 17, no. 10, pp. 1237-1239, 2001.

[25] Y. C. Hou, S. Y. Tsai, P. Y. Lai, Y. S. Chen, and P. D. L. Chao, "Metabolism and pharmacokinetics of genipin and geniposide in rats," Food and Chemical Toxicology, vol. 46, no. 8, pp. 2764-2769, 2008.

[26] X. Zheng, X.-T. Huang, N. Li et al., "Determination of geniposidic acid in rat plasma by LC-MS/MS and its application to in vivo pharmacokinetic studies," Journal of Chromatography B, vol. 887-888, pp. 138-142, 2012.

[27] L.-H. He, J. Li, Y.-X. Deng, X.-J. Zhang, R. Chen, and Y. Lv, "Comparative investigation on the pharmacokinetics of geniposide in type 2 diabetic and normal rats after oral administration of Fructus Gradeniae extract," Journal of Chromatography B, vol. 1033-1034, pp. 180-186, 2016.

[28] X. J. Zhang, S. Liu, Z. F. Pi, Z. Q. Liu, and F. R. Song, "Simultaneous quantification method for comparative pharmacokinetics studies of two major metabolites from geniposide and genipin by online mircrodialysis-UPLC-MS/MS," Journal of Chromatography B, vol. 1041-1042, pp. 11-18, 2017.

[29] H. L. Wang, J. Li, X.-M. Gao, and Y.-X. Chang, "Ionic liquids vortex-simplified matrix solid phase dispersion for simultaneous determination of terpenoids, crocins, quinic acid derivatives and flavonoids in Gardeniae fructus by ultraperformance liquid chromatography," Journal of Separation Science, vol. 42, no. 10, pp. 1886-1895, 2019.

[30] Y. Zhang, F. Fei, L. Zhen et al., "Sensitive analysis and simultaneous assessment of pharmacokinetic properties of crocin and crocetin after oral administration in rats," Journal of Chromatography, vol. 1044-1045, pp. 1-7, 2017. 